\title{
Front Matter: Volume 7692
}

, "Front Matter: Volume 7692," Proc. SPIE 7692, Unmanned Systems Technology XII, 769201 (19 May 2010); doi: 10.1117/12.864061

SPIE Event: SPIE Defense, Security, and Sensing, 2010, Orlando, Florida, United SPIE. States 


\title{
PROCEEDINGS OF SPIE
}

\section{Unmanned Systems Technology XII}

\author{
Grant R. Gerhart \\ Douglas W. Gage \\ Charles M. Shoemaker \\ Editors
}

6-9 April 2010

Orlando, Florida, United States

Sponsored and Published by

SPIE 
The papers included in this volume were part of the technical conference cited on the cover and title page. Papers were selected and subject to review by the editors and conference program committee. Some conference presentations may not be available for publication. The papers published in these proceedings reflect the work and thoughts of the authors and are published herein as submitted. The publisher is not responsible for the validity of the information or for any outcomes resulting from reliance thereon.

Please use the following format to cite material from this book:

Author(s), "Title of Paper," in Unmanned Systems Technology XII, edited by Grant R. Gerhart, Douglas W. Gage, Charles M. Shoemaker, Proceedings of SPIE Vol. 7692 (SPIE, Bellingham, WA, 2010) Article CID Number.

ISSN 0277-786X

ISBN 9780819481566

Published by

SPIE

P.O. Box 10, Bellingham, Washington 98227-0010 USA

Telephone +1 3606763290 (Pacific Time) · Fax +1 3606471445

SPIE.org

Copyright (C) 2010, Society of Photo-Optical Instrumentation Engineers

Copying of material in this book for internal or personal use, or for the internal or personal use of specific clients, beyond the fair use provisions granted by the U.S. Copyright Law is authorized by SPIE subject to payment of copying fees. The Transactional Reporting Service base fee for this volume is $\$ 18.00$ per article (or portion thereof), which should be paid directly to the Copyright Clearance Center (CCC), 222 Rosewood Drive, Danvers, MA 01923. Payment may also be made electronically through CCC Online at copyright.com. Other copying for republication, resale, advertising or promotion, or any form of systematic or multiple reproduction of any material in this book is prohibited except with permission in writing from the publisher. The CCC fee code is 0277-786X/10/\$18.00.

Printed in the United States of America.

Publication of record for individual papers is online in the SPIE Digital Library.

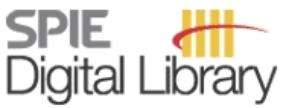

SPIEDigitalLibrary.org

Paper Numbering: Proceedings of SPIE follow an e-First publication model, with papers published first online and then in print and on CD-ROM. Papers are published as they are submitted and meet publication criteria. A unique, consistent, permanent citation identifier (CID) number is assigned to each article at the time of the first publication. Utilization of CIDs allows articles to be fully citable as soon they are published online, and connects the same identifier to all online, print, and electronic versions of the publication. SPIE uses a six-digit CID article numbering system in which:

- The first four digits correspond to the SPIE volume number.

- The last two digits indicate publication order within the volume using a Base 36 numbering system employing both numerals and letters. These two-number sets start with 00, 01, 02, 03, 04, $05,06,07,08,09,0 A, 0 B \ldots 0 Z$, followed by 10-1Z, 20-2Z, etc.

The CID number appears on each page of the manuscript. The complete citation is used on the first page, and an abbreviated version on subsequent pages. Numbers in the index correspond to the last two digits of the six-digit CID number. 


\section{Contents}

ix Conference Committee

SELF-ORGANIZING, COLLABORATIVE, AND UNMANNED ISR ROBOTS: JOINT SESSION WITH CONFERENCE 7707

769202 All weather collision avoidance for unmanned aircraft systems [7692-15]

M. Contarino, Scire Consultants LLC (United States)

769203 Lidar-based door and stair detection from a mobile robot [7692-02]

M. Bansal, B. Southall, B. Matei, J. Eledath, H. Sawhney, Sarnoff Corp. (United States)

HUMAN ROBOT INTERACTION (HRI)

769204 Human-robot interaction research for current and future military applications: from the laboratory to the field [7692-03]

K. A. Cosenzo, M. J. Barnes, U.S. Army Research Lab. (United States)

769205 Robust natural language dialogues for instruction tasks [7692-04]

M. Scheutz, Indiana Univ. (United States)

769206 Field testing of tele-operation versus shared and traded control for military assets: an evaluation involving real-time embedded simulation and soldier assessment [7692-05] J. S. Metcalfe, DCS Corp. (United States); J. Alban, U.S. Army Tank-Automotive Research, Development and Engineering Ctr. (United States); K. Cosenzo, U.S. Army Research Lab. (United States); T. Johnson, E. Capstick, DCS Corp. (United States)

769207 Comparison of tele-operation and supervisory control for navigation and driving with degraded communications [7692-06]

G. Witus, Turing Associates, Inc. (United States); R. D. Ellis, Wayne State Univ. (United States); R. Karlsen, U.S. Army Tank Automotive Research, Development and Engineering Ctr. (United States); S. Hunt, Turing Associates, Inc. (United States)

769208 Combined virtual and real robotic test-bed for single operator control of multiple robots [7692-07]

S. Y.-S. Lee, S. Hunt, A. Cao, A. Pandya, Wayne State Univ. (United States)

769209 Experimentation and evaluation of threat detection and local area awareness using advanced computational technologies in a simulated military environment [7692-08] J. S. Metcalfe, DCS Corp. (United States); G. Brick Larkin, U.S. Army Research Lab. (United States); T. Johnson, DCS Corp. (United States); K. Oie, U.S. Army Research Lab. (United States); V. Paul, U.S. Army Tank-Automotive Research, Development and Engineering Ctr. (United States); J. Davis, U.S. Army Research Lab. (United States) 
$76920 \mathrm{~A}$ Human-robot interaction modeling and simulation of supervisory control and situational awareness during field experimentation with military manned and unmanned ground vehicles [7692-10]

T. Johnson, J. Metcalfe, B. Brewster, C. Manteuffel, M. Jaswa, DCS Corp. (United States); T. Tierney, U.S. Army Tank-Automotive Research, Development and Engineering Ctr. (United States)

7692 OB Delegation control of multiple unmanned systems [7692-11]

S. R. Flaherty, R. J. Shively, U.S. Army Aeroflightdynamics Directorate (United States)

\section{PERCEPTION}

7692 0C Stereo-vision-based perception capabilities developed during the Robotics Collaborative Technology Alliances program [7692-12]

A. Rankin, M. Bajracharya, A. Huertas, A. Howard, B. Moghaddam, S. Brennan, A. Ansar, B. Tang, M. Turmon, L. Matthies, Jet Propulsion Lab. (United States)

7692 OD Velodyne HDL-64E lidar for unmanned surface vehicle obstacle detection [7692-14] R. Halterman, M. Bruch, Space and Naval Warfare Systems Ctr. Pacific (United States)

7692 OE Close range ISR (PRISTA) and close quarters combat (CQC) with unmanned aerial systems (UAS) [7692-01]

J. Maynell, Lite Machines Corp. (United States)

7692 OF Combining structure and appearance cues for real-time pedestrian detection [7692-16] M. Bansal, S.-H. Jung, B. Matei, J. Eledath, H. Sawhney, Sarnoff Corp. (United States)

7692 OG ESARR: enhanced situational awareness via road sign recognition [7692-17] V. E. Perlin, D. B. Johnson, M. M. Rohde, R. M. Lupa, Quantum Signal LLC (United States); G. Fiorani, S. Mohammad, U.S. Army Tank-Automotive Research, Development and Engineering Ctr. (United States)

$7692 \mathrm{OH}$ A final evaluation of pedestrian detection and tracking from a moving platform [7692-18] B. A. Bodt, U.S. Army Research Lab. (United States); R. Camden, MPRI, a division of L3 Services Group (United States)

$769201 \quad$ Vision systems for manned and robotic ground vehicles [7692-19] J. N. Sanders-Reed, Boeing-SVS, Inc. (United States); P. L. Koon, The Boeing Co. (United States)

7692 0J 3D vision upgrade kit for TALON robot [7692-20] R. Edmondson, J. Vaden, B. Hyatt, J. Morris, J. L. Pezzaniti, D. B. Chenault, Polaris Sensor Technologies, Inc. (United States); J. Tchon, T. Barnidge, Rockwell Collins, Inc. (United States); S. Kaufman, Foster-Miller, Inc. (United States); B. Pettijohn, U.S. Army Research Lab. (United States)

7692 OK Defecting and tracking moving objects from a moving plafform using epipolar constraints [7692-21]

J. C. McBride, A. Ostapchenko, Charles River Analytics, Inc. (United States); H. Schultz, Univ. of Massachusetts (United States); M. S. Snorrason, Charles River Analytics, Inc. (United States) 
7692 OL Cognitive object recognition system (CORS) [7692-22]

C. Raju, K. M. Varadarajan, N. Krishnamurthi, S. XU, UtopiaCompression Corp. (United States); I. Biederman, The Univ. of Southern California (United States); T. Kelley, U.S. Army Research Lab. (United States)

\section{SPECIAL TOPICS}

7692 OM Unmanned systems to support the human exploration of Mars [7692-23]

D. W. Gage, XPM Technologies (United States)

$76920 \mathrm{~N} \quad$ Automatic payload deployment system [7692-24]

N. Pezeshkian, H. G. Nguyen, A. Burmeister, K. Holz, A. Hart, Space and Naval Warfare Systems Ctr. Pacific (United States)

769200 Fusing ultra-wideband radar and lidar for small UGV navigation in all-weather conditions [7692-25]

B. Yamauchi, iRobot Corp. (United States)

7692 OP Experimental evaluation of assistive behaviors for man-portable robots [7692-26]

C. Pierce, D. Baran, B. Bodt, U.S. Army Research Lab. (United States)

\section{INTELLIGENT BEHAVIORS}

$7692 \mathrm{OR} \quad$ Experiments with autonomous mobile radios for wireless tethering in tunnels [7692-28] K. L. Moore, M. D. Weiss, J. P. Steele, K. Anderson, J. Hulbert, C. Karlsson, E. Larson, C. Meehan, A. Weinstein, Colorado School of Mines (United States)

7692 OS Q-learning approach to automated unmanned air vehicle (UAV) demining [7692-29] S. Ferrari, G. Daugherty, Duke Univ. (United States)

7692 OT Unmanned aircraft systems as wingmen [7692-30]

R. Garcia, Motile Robotics Inc. (United States); L. Barnes, The Univ. of Texas at Arlington (United States); M. Fields, U.S. Army Research Lab. (United States)

7692 OU Fault tolerant formation control of nonholonomic mobile robots using online approximators [7692-31]

B. T. Thumati, T. A. Dierks, S. Jagannathan, Missouri Univ. of Science and Technology (United States)

7692 OV Control of an indoor autonomous mobile communications relay via antenna diversity [7692-32]

B. Griffin, R. Fierro, I. Palunko, The Univ. of New Mexico (United States)

7692 OW Online gaming for learning optimal team strategies in real time [7692-33]

G. Hudas, U.S. Army Tank-Automotive Research, Development and Engineering Ctr. (United States); F. L. Lewis, K. G. Vamvoudakis, The Univ. of Texas at Arlington (United States) 
$76920 Z$ Urban Hopper [7692-36]

J. R. Salton, S. Buerger, L. Marron, J. Feddema, G. Fischer, C. Little, B. Spletzer, P. Xavier, Sandia National Labs. (United States); A. A. Rizzi, M. P. Murphy, J. Giarratana,

M. D. Malchano, C. A. Weagle, Boston Dynamics (United States)

$769211 \quad$ Agile and dexterous robot for inspection and EOD operations [7692-38]

D. A. Handelman, G. H. Franken, H. Komsuoglu, American Android Corp. (United States)

769212 Toward humanoid robots for operations in complex urban environments [7692-39]

J. E. Pratt, P. Neuhaus, M. Johnson, J. Carff, B. Krupp, Institute for Human and Machine

Cognition (United States)

769213 On-board SLAM for indoor UAV using a laser range finder [7692-40]

M. Alpen, C. Willrodt, K. Frick, J. Horn, Helmut-Schmidt-Univ. (Germany)

769214 Optimal powering schemes for legged robotics [7692-41]

P. Muench, D. Bednarz, G. P. Czerniak, U.S. Army Tank-Automotive Research, Development and Engineering Ctr. (United States); K. C. Cheok, Oakland Univ. (United States)

$769215 \quad$ R-Gator: an unmanned utility vehicle [7692-42]

S. J. Moorehead, Deere \& Co. (United States); C. K. Wellington, Carnegie Mellon Univ.

(United States); H. Paulino, J. F. Reid, Deere \& Co. (United States)

\section{GOVERNMENT PROGRAMS}

769217 Life after Future Combat System: a family of ground robotic systems [7692-44]

D. G. Knichel, Maneuver Support Ctr. (United States)

769218 Integration of a high degree of freedom robotic manipulator on a large unmanned ground vehicle [7692-45]

J. Giesbrecht, B. Fairbrother, J. Collier, B. Beckman, Defence Research and Development Canada (Canada)

7692 1B An overview of the 2009 Fort Hood Robotics Rodeo [7692-48]

S. Norberg, U.S. Army Tank Automotive Research, Development and Engineering Ctr. (United States)

7692 1C Semi-autonomous UAV/UGV for dismounted urban operations [7692-49]

M. Trentini, B. Beckman, Defence Research and Development Canada (Canada)

7692 1D Fractionated multirobotics: a hierarchical approach [7692-50]

G. Konesky, K-Plasma, Ltd. (United States) 
7692 IE Human leader and robot follower team: correcting leader's position from follower's heading [7692-51]

J. Borenstein, D. Thomas, Univ. of Michigan (United States); B. Sights, Space and Naval Warfare Systems Ctr. Pacific (United States); L. Ojeda, Univ. of Michigan (United States); P. Bankole, D. Fellars, Space and Naval Warfare Systems Ctr. Pacific (United States)

7692 IG Robotic Mounted Detection System: robotics for route clearance [7692-53] J. Hutchisonl, G. Klager, U.S. Army Night Vision \& Electronic Sensors Directorate (United States); E. McCoy, BRTRC (United States); D. Fite, B. Frederick, General Dynamics Robotic Systems (United States)

$769211 \quad$ A visual odometry method based on the SwissRanger SR4000 [7692-55]

C. Ye, Univ. of Arkansas at Little Rock (United States); M. Bruch, Space and Naval Warfare Systems Ctr. Pacific (United States)

\section{POSTER SESSION}

7692 1L Device capable small arms ammunition for unmanned systems [7692-60]

N. P. Bergeron, J. W. Sweeney, C. G. Wilson, Lovisiana Tech Univ. (United States)

$76921 \mathrm{M} \quad$ Pressurized structures-based hybrid unmanned aerial vehicles [7692-61]

H. L. Edge, A. Brown, U.S. Army Research Lab. (United States); J. Collins, Motile Robotics Inc. (United States)

7692 iN A reusable robotic grasping creator [7692-62]

Y. Li, J. Keesling, C. Pholsiri, N. Tardella, J. English, Energid Technologies Corp. (United States)

7692 IR Heuristics-enhanced dead-reckoning (HEDR) for accurate position tracking of teleoperated UGVs [7692-69]

J. Borenstein, A. Borrell, R. Miller, D. Thomas, Univ. of Michigan (United States)

7692 is Monocular panoramic 3D reconstruction based on a particle filter [7692-70]

F. Pagel, Fraunhofer Institute for Optronics, System Technologies and Image Exploitation (Germany)

7692 IT Exploiting uncalibrated stereo on a UAV platform [7692-71]

M. Banish, M. Rodgers, B. Hyatt, R. Edmondson, D. B. Chenault, Polaris Sensor Technologies, Inc. (United States); J. Heym, Consultant (United States); P. DiNardo, B. Gruber, J. Johnson, K. Dobson, U.S. Army Aviation and Missle Research, Development and Engineering Ctr. (United States)

$76921 \mathrm{X}$ Cooperative energy harvesting for long-endurance autonomous vehicle teams [7692-77] S. F. Page, J. D. Rogers, K. May, D. R. Myatt, D. Hickman, M. I. Smith, Waterfall Solutions Ltd. (United Kingdom)

$76921 Y$ On the reliability of collaboration and coordination of unmanned vehicle network [7692-78] A. M. Dixit, K. Saab, H. Singh, Wayne State Univ. (United States) 
769212 Multichannel, agile, computationally enhanced camera based on the PANOPTES architecture [7692-79]

P. Milojkovic, U.S. Army Research Lab. (United States); J. Gill, D. Frattin, K. Coyle, K. Haack, S. Myhr, Northrop Grumman Information Systems (United States); D. Rajan, S. Douglas,

P. Papamichalis, M. Somayaji, M. Christensen, Southern Methodist Univ. (United States);

K. Krapels, U.S. Army Night Vision \& Electronic Sensors Directorate (United States)

Author Index 


\title{
Conference Committee
}

\author{
Symposium Chair
}

Michael T. Eismann, Air Force Research Laboratory (United States)

Symposium Cochair

William Jeffrey, HRL Laboratories, LLC (United States)

Conference Chairs

Grant R. Gerhart, U.S. Army Tank-Automotive Research, Development and Engineering Center (United States)

Douglas W. Gage, XPM Technologies (United States)

Charles M. Shoemaker, General Dynamics Robotic Systems (United States)

Program Committee

Stephen Balakirsky, National Institute of Standards and Technology (United States)

Johann Borenstein, University of Michigan (United States)

Jonathan A. Bornstein, Army Research Laboratory (United States)

Bruce L. Digney, Defence Research and Development Canada (Canada)

Rajiv V. Dubey, University of South Florida (United States)

Hobart R. Everett, Space and Naval Warfare Systems Center Pacific (United States)

Scott Fish, The University of Texas at Austin (United States)

David J. Gorsich, U.S. Army Tank-Automotive Research, Development and Engineering Center (United States)

Susan G. Hill, U.S. Army Research Laboratory (United States)

Karl D. lagnemma, Massachusetts Institute of Technology (United States)

Jeffrey F. Jaster, U.S. Army Tank-Automotive Research, Development and Engineering Center (United States)

Gene A. Klager, U.S. Army Night Vision \& Electronic Sensors Directorate (United States)

Andreas F. Koschan, The University of Tennessee (United States)

James H. Lever, U.S. Army Corps of Engineers (United States)

Larry H. Matthies, Jet Propulsion Laboratory (United States)

Kevin L. Moore, Colorado School of Mines (United States) 
Hoa G. Nguyen, Space and Naval Warfare Systems Center Pacific (United States)

James L. Overholt, U.S. Army Tank-Automotive Research, Development and Engineering Center (United States)

Marc Raibert, Boston Dynamics (United States)

Klaus-Juergen Schilling, Julius-Maximilians-Universität Würzburg (Germany)

Nahid N. Sidki, SAIC (United States)

Harpreet Singh, Wayne State University (United States)

Magnus S. Snorrason, Charles River Analytics, Inc. (United States)

Anthony Stentz, Carnegie Mellon University (United States)

David L. Stone, Mechatron Consulting (United States)

Venkataraman Sundareswaran, Teledyne Scientific Company (United States)

Brian H. Wilcox, Jet Propulsion Laboratory (United States)

Gary Witus, Turing Associates, Inc. (United States)

Brian M. Yamauchi, iRobot Corporation (United States)

\section{Session Chairs}

1 Self-Organizing, Collaborative, and Unmanned ISR Robots: Joint Session with Conference 7707

Douglas W. Gage, XPM Technologies (United States)

Melanie Dumas, Defense Advanced Research Projects Agency (United States)

$2 \quad$ Human Robot Interaction (HRI)

Susan G. Hill, U.S. Army Research Laboratory (United States)

Gary Witus, Turing Associates, Inc. (United States)

3 Perception

Magnus S. Snorrason, Charles River Analytics, Inc. (United States)

Larry H. Matthies, Jet Propulsion Laboratory (United States)

$4 \quad$ MAST I: Joint Session with Conference 7679

Joseph N. Mait, U.S. Army Research Laboratory (United States)

$5 \quad$ MAST II: Joint Session with Conference 7679

Joseph N. Mait, U.S. Army Research Laboratory (United States)

6 Special Topics

Stuart H. Young, U.S. Army Research Laboratory (United States)

Brian M. Yamauchi, iRobot Corporation (United States)

Robert E. Karlsen, U.S. Army Tank-Automotive Research, Development and Engineering Center (United States) 
$7 \quad$ Intelligent Behaviors

Frank L. Lewis, The University of Texas at Arlington (United States)

Gregory R. Hudas, U.S. Army Tank-Automotive Research, Development and Engineering Center (United States)

8 Mobility

Paul L. Muench, U.S. Army Tank-Automotive Research, Development and Engineering Center (United States)

Michael R. Perschbacher, RovnoTech (United States)

9 Government Programs

Jeffrey F. Jaster, U.S. Army Tank-Automotive Research, Development and Engineering Center (United States)

Jared Giesbrecht, Defence Research and Development Canada (Canada)

10 Navigation

Charles M. Shoemaker, General Dynamics Robotic Systems (United States)

Mel W. Torrie, Autonomous Solutions, Inc. (United States) 
Downloaded From: https://www.spiedigitallibrary.org/conference-proceedings-of-spie on 26 Apr 2023

Terms of Use: https://www.spiedigitallibrary.org/terms-of-use 